\title{
Monitoring in non-traumatic coma. Part II: electroencephalography
}

\author{
R C TASKER, S BOYD, A HARDEN, AND D J MATTHEW \\ Hospital for Sick Children, Great Ormond Street, London
}

SUMmARY Forty eight comatose children had electroencephalograms (EEG) recorded during the acute phase of their illnesses. These were classified according to a simple grading system and the findings correlated with the presence of seizures, deep coma, minimum cerebral perfusion pressure, and eventual neurological outcome. Serial EEGs proved important, particularly when slow activity was seen initially. None of the 20 patients who showed low amplitude EEG activity or electrocerebral silence at any stage of the acute illness did well. Discharges were seen in only 13 of the 29 patients with seizures and their presence did not correlate with outcome except in five patients with a distinctive pattern of discharges, none of whom had a good outcome. EEG findings associated with poor outcome did not always correlate with the clinical assessment of deep coma, emphasising the difficulties of neurological evaluation in these patients. Five of the patients with cerebral perfusion pressures greater than $42 \mathrm{~mm} \mathrm{Hg}$ had a poor outcome that was predicted by serial EEGs. In nine patients with a minimum cerebral perfusion pressure in the borderline range $38-42 \mathrm{~mm} \mathrm{Hg}$ the EEG was useful as an indication of the outcome.

The EEG reflects changes in cerebral function which may be due to multifactorial or repeated insults. An EEG is therefore important in both the initial assessment and as an indicator of the neurological outcome, particularly in those patients in whom the cerebral perfusion pressure has apparently been adequate or within the borderline range.

Clinical findings in comatose patients whose intracranial pressure is being monitored are influenced by the underlying neurological condition and by drug treatment. The minimum cerebral perfusion pressure measured during the acute illness, although useful, is only a limited guide to prognosis because it gives no indication of the severity of the preceding cerebral damage or of changes of cerebral function during monitoring. ${ }^{1}$

In children with acute non-traumatic cerebral damage, the electroencephalogram (EEG) provides information about the severity and distribution of altered function of the cerebral cortex, presence of discharges, and even (on occasion), a clue to the possible aetiology. In Reye's syndrome, ${ }^{2}$ near drowning, ${ }^{3}$ and after cardiac arrest ${ }^{4}$ the EEG taken at an early stage was of practical help in assessing the final neurological outcome. As only nine of the 49 children already reviewed ${ }^{1}$ presented with one of these three conditions, we have asessed the contribution of the EEG findings to the assessment of prognosis in this larger more heterogeneous group of comatose children. Particular attention was paid to the association between intracranial measurements and EEG features.

\section{Patients and methods}

Forty eight of the 49 children previously reported ${ }^{1}$ had EEGs recorded in the paediatric intensive care unit during the acute phase of their illness. All EEGs were recorded at the bedside using Grass 8 channel EEG machines and previously described techniques. ${ }^{4}$ The EEGs were usually recorded within 24 hours of admission, and no longer than 36 hours in any patient. One hundred and sixty two EEGs were carried out on the 48 patients during 351 patient days in hospital. Eight children had only one EEG, but serial EEGs were taken in most (two in 13 , three in 9 , and four or more in 18 children).

The EEGs were graded in order of severity into one of the four categories defined in part $I .{ }^{1}$ This grading was adapted from systems used previously in patients with Reye's syndrome, ${ }^{2}$ near drowning, ${ }^{3}$ and after cardiac arrest, ${ }^{4}$ and like these was based mainly on the amplitude of the predominant activity. 
In addition to this, however, those EEGs showing discharges were given one of three subscripts indicating the type of discharges. In nine patients receiving thiopentone the EEG grades of II (low amplitude $<50 \mu \mathrm{V}$ ) or III (isoelectric) were not included in the data analysis. In these cases the EEG grades before the drug was given and after the drug had cleared were used. Attempts to stimulate the patients were made during each recording but environmental conditions made this difficult to assess and so it has not been included in this study. Although the recordings were reviewed retrospectively there was little discrepancy between the original report and the subsequent grading.

Clinical findings including seizures in the 24 hour period before or after admission, and deep coma (fixed dilated pupils, or a Glasgow coma score of five or less not attributable to drugs) on admission, were noted. Maximum intracranial pressure and minimum cerebral perfusion pressure together with the neurological outcome ${ }^{1}$ were correlated with EEG grading scores.

The $\chi^{2}$ test with Yates's correction was used to assess the differences between proportions.

\section{Results}

The diagnosis, neurological outcome, and EEG findings were listed previously. ${ }^{1}$ The association between the initial EEG and the eventual outcome is shown in table 1 . In five patients (cases 5, 24, 31, $37,48^{1}$ ) the initial EEG could not be graded for background activity because of the presence of continuous discharges.

All nine patients whose initial EEG showed low amplitude activity (grade II) or electrocerebral silence (grade III) had a poor neurological outcome. Nineteen of the 34 patients in whom the initial EEG was either normal or showed slow activity (grade I) did well; the remainder had either moderate $(n=4)$, or poor $(n=11)$ outcomes.

In the 40 children who had serial EEGs the worst EEG grade was correlated with outcome (table 2). None of the 20 patients with either grade II or

Table 1 Initial EEG grade in relation to eventual neurological outcome $(n=43)$

\begin{tabular}{lclr}
\hline Initial grade & \multicolumn{2}{l}{ Outcome } & \\
\cline { 2 - 4 } & Good & Moderate & Poor \\
\hline Normal & 5 & 2 & 0 \\
I & 14 & 2 & 11 \\
II & 0 & 0 & 5 \\
III & 0 & 0 & 4 \\
\hline
\end{tabular}

Table 2 Worst EEG grade in the 40 patients with serial studies in relation to eventual outcome

\begin{tabular}{lclc}
\hline Worst grade & \multicolumn{2}{l}{ Outcome } & \\
\cline { 2 - 4 } & Good & Moderate & Poor \\
\hline Normal & 0 & 0 & 0 \\
I & 15 & 2 & 3 \\
II & 0 & 1 & 7 \\
III & 0 & 0 & 12 \\
\hline
\end{tabular}

grade III at any stage did well. Indeed, 19 of these 20 patients had poor outcomes. Fifteen of the 20 patients with grade I as their worst grading did well, but two had moderate, and three had poor outcomes. The association between grade II or III EEG and poor outcome was highly significant $(p<0 \cdot 001)$.

The five children (cases $7,15,28,32,37$ ) who had either moderate or poor outcomes despite grade I EEGs had additional EEG features that were not present in those who did well. In three children (cases 15, 28, 32) there was no clinical or EEG improvement 9-12 days after the initial insult (fig 1). This is in pronounced contrast to the patients who did well, in whom the grade I pattern only was seen for a maximum of six days before a clinical improvement in the level of consciousness was noted. Cases 7 and 37 had focal low amplitude activity, and case 37 also had seizures that were difficult to control, with 'electrical storms' being recorded at presentation and 36 hours later. Although brief episodes of low amplitude activity occurring either spontaneously or in response to stimuli were a prominent feature in the EEGs of case 15 , similar features were present though less pronounced in some patients who did well.

Twenty nine patients had clinical seizures in the 24 hours before or after admission; only 13 of these had discharges in the initial record and their outcomes are compared with those of patients without discharges in table 3 . There was no difference in outcome between patients with or without discharges except that none of the five patients with 'electrical storms' in the initial EEG recording (fig 2) did well, and the clinical seizures in these five patients were difficult to control.

Of the 12 patients presenting in deep coma, two initially showed electrocerebral silence (grade III). Low amplitude background activity (grade II) was seen in a further three patients. Slow activity (grade I) was present in five patients, and two could not be graded because of continuous 'electrical storms'. Review of the worst EEGs in these children showed that seven had grade III, and four others had grade II tracings. The EEG in the twelfth 

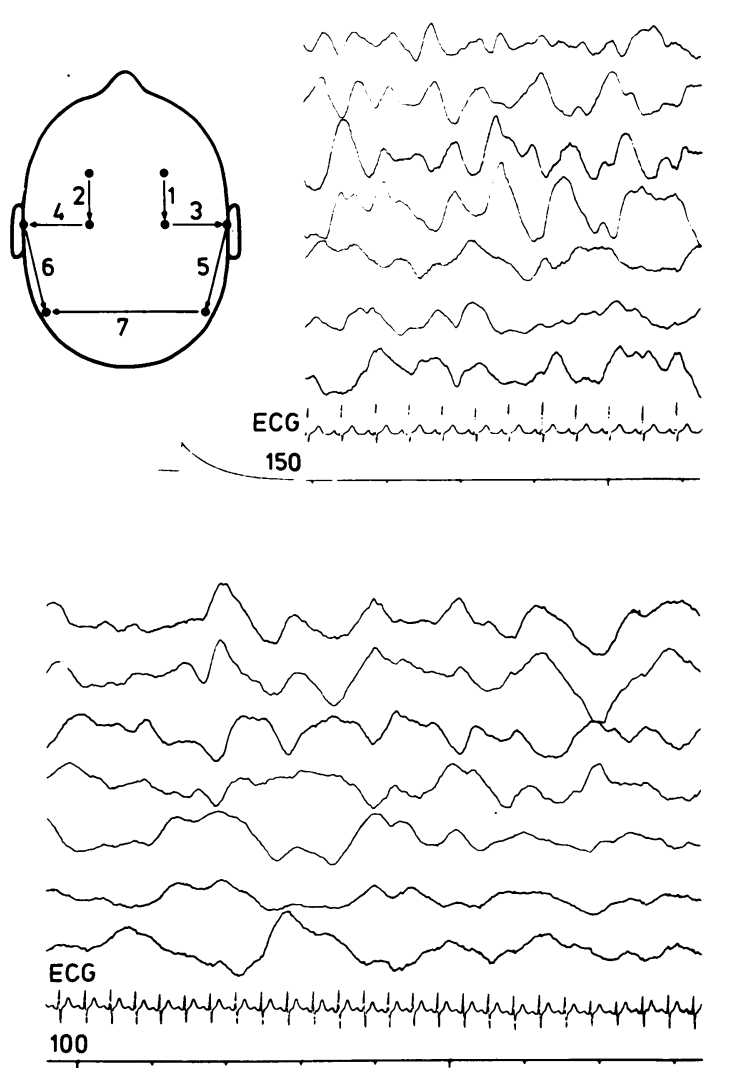

Fig 1 An infant aged 18 months with a metabolic encephalopathy (case 28) had generalised large amplitude slow activities (grade I) on initial EEG (upper trace). Nine days later (lower trace) slow activity is even more prominent though still grade I. He had adequate cerebral perfusion pressure throughout and survived with a moderate handicap.

Table 3 Relation between discharges in the initial EEG and eventual outcome

\begin{tabular}{lllc}
\hline Discharges & \multicolumn{2}{l}{ Outcome } & \\
\cline { 2 - 4 } & Good & Moderate & Poor \\
\hline None & 17 & 2 & 16 \\
Continuous (a) & 1 & 0 & 0 \\
Isolated (b) & 2 & 2 & 3 \\
'Electrical storms' (c) & 0 & 1 & 4 \\
\hline
\end{tabular}

patient (case 32), although grade I, never improved. In addition two patients (cases 8 and 45) with grade III, and two (cases 40 and 46 ) with grade II, initial EEGs were not deeply comatose. In the two cases with grade III tracings, muscle relaxants and cycloplegics had been given shortly before admission.
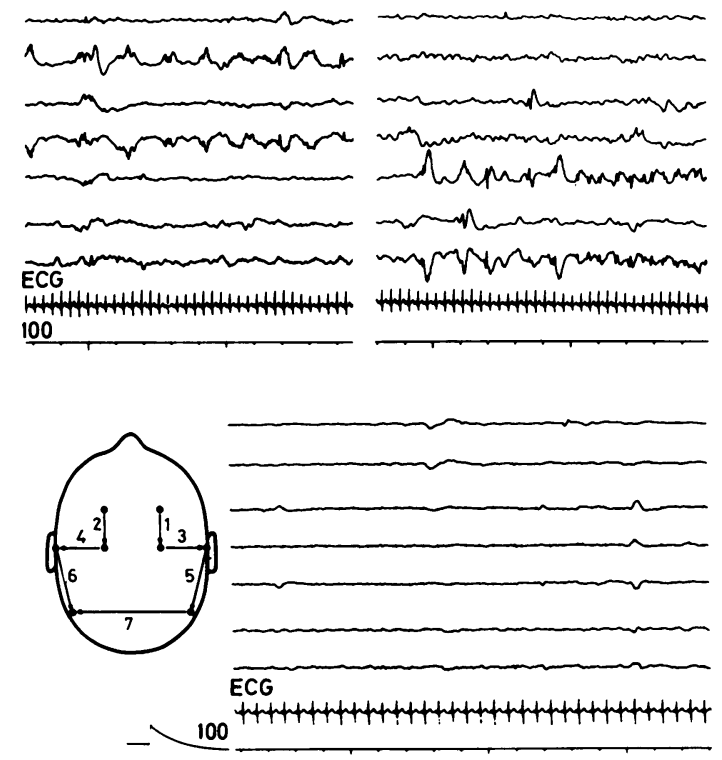

Fig 2 An infant aged 6 months with Reye's syndrome after chickenpox (case 31) showed an unusual EEG picture; runs of discharges shifting in distribution and changing in both rate and morphology ('electrical storms') were seen in the initial EEG (upper traces). He had seizures that were difficult to control. EEG tracings gradually deteriorated and 14 days later were of low amplitude (grade II) (lower trace). He survived with severe neurological impairment.

The worst EEG was grade II or III in all nine patients with minimum cerebral perfusion pressure less than $38 \mathrm{~mm} \mathrm{Hg}$, and they all had poor outcomes. ${ }^{1}$

There were 20 patients with serial EEGs who had minimum cerebral perfusion pressure greater than $42 \mathrm{~mm} \mathrm{Hg}$, and their outcome is shown in table 4 . Although most remained no worse than grade I (with a good outcome), there were seven patients (cases $1,32,33,35,40,47$, and 49) with poor neurological outcomes. ${ }^{1}$ Five of these had EEGs of grade II or III, and of the other two children case 32 (although grade I throughout) never improved, and case 1 did not have serial investigations.

Table 4 Worst EEG grade (serial studies) in 20 patients with adequate cerebral perfusion pressure $(>42 \mathrm{~mm} \mathrm{Hg})$

\begin{tabular}{llll}
\hline Worst grade & \multicolumn{2}{l}{ Outcome } & \\
\cline { 2 - 4 } & Good & Moderate & Poor \\
\hline I & 13 & 1 & 1 \\
II & 0 & 0 & 2 \\
III & 0 & 0 & 3 \\
\hline
\end{tabular}


Table 5 Worst EGG grade (serial studies) in relation to outcome in seven patients with cerebral perfusion pressures of $38-42 \mathrm{~mm} \mathrm{Hg}$

\begin{tabular}{llll}
\hline Worst grade & \multicolumn{2}{l}{ Outcome } & \\
\cline { 2 - 4 } & Good & Moderate & Poor \\
\hline I & 2 & 1 & 1 \\
II & 0 & 1 & 0 \\
III & 0 & 0 & 2 \\
\hline
\end{tabular}

In the nine children whose minimum cerebral perfusion pressure was in the borderline range $(38-42 \mathrm{~mm} \mathrm{Hg})$ the outcomes were equally distributed among the three groups; three were good, three moderate, and three poor. The worst EEG in relation to outcome in the seven of them who had serial studies is shown in table 5. Two of the three patients (cases 26 and 39) with poor outcomes had grade III tracings (electrocerebral silence). The third patient (case 15) had brief episodes of low amplitude activity (but was classified as grade I). Two of the three patients (cases 9 and 14) with good outcomes had serial EEGs and they remained grade I. Two of the three patients with moderate outcomes had serial EEGs and the worst grade was II in case 4, and $I$ in case 37. In addition, in the latter case 'electrical storms' had initially been recorded.

Of the five patients in whom intracranial pressure monitoring failed, three of the four with poor outcomes showed grade II or III tracings. The fourth patient (case 7) had focal low amplitude activity.

\section{Discussion}

There are obvious advantages in grading EEG features that may help predict the neurological outcome of a comatose child. The grading used in this series (based mainly on amplitude) was found useful by other authors in specific types of encephalopathy. ${ }^{2-4}$ Our results suggest that the grading is valuable in a wider context. All nine patients whose initial EEG showed grade II (low amplitude) or grade III (electrocerebral silence) tracings had poor outcomes, as did 19 of the 20 children who had grade II or III tracings at any stage during their illnesses (excluding EEGs taken when intravenous thiopentone had been given). Only one child (case 4) with a grade II tracing had a moderate neurological outcome. There was never any improvement in the EEGs of patients initially showing either grade II or III tracings.

Moderate to large amplitude ( $0 \cdot 5-3$ cycles/second) slow activity (grade I) is the usual EEG change associated with any acute encephalopathy or encephalitis and represents a potentially reversible alteration of cerebral function, and it is important to establish this in the early phase of neurointensive care. During the acute phase of the illness, however, further damage is likely to occur, so serial EEG studies are important when grade I is seen initially; eight of our 23 patients later deteriorated to grade II or III. Only 15 of the 20 patients who never had an EEG worse than grade I had a good outcome. Serial studies in the other five showed unusually persistent slow activities without any increase in frequency in three cases, contrasting with the gradual increase in frequency of EEG activities usually seen during recovery. Although less than five second periods of extremely low amplitude (either spontaneously or in response to stimuli) occurred in one patient classified as grade I who had a poor outcome, this EEG feature alone does not seem to have any discriminatory value, as it was noted in some children who recovered fully.

Of the five patients whose initial EEG could not be graded into one of the four categories because discharges were their main feature, one showed clinical and EEG evidence of status epilepticus that was reversible. The 'electrical storms' seen in the other four patients are a distinctive EEG pattern that is commonly associated with damage to the cortical microcirculation and may account for the outcome in these patients. Any neurological sequelae seem to be related to the severity and extent of this underlying process, and four of our five patients who showed 'electrical storms' (one of whom was also initially graded as II) were eventually classified as grade II. Neither the presence of seizures nor of isolated discharges were in themselves pointers to eventual outcome, though repetitive stereotyped discharges (not seen in any of these patients) are highly suggestive of herpes simplex encephalitis. Localised EEG abnormalities, whether discharges or areas of low amplitude, were usually associated with focal neurological signs.

All the patients diagnosed on admission as deeply comatose had poor outcomes ${ }^{1}$ and most of these had grade II or III EEG tracings at some time. It was, however, surprising that the EEG was grade I initially in five patients. In addition, two patients with grade III EEG tracings on presentation were not clinically identified as being deeply comatose. Neurological assessment is, however, difficult in sedated and paralysed patients and the EEG may be the best method of assessing cerebral function, though the effects of drugs (such as barbiturates) should be taken into acccount.

The finding that seven patients with no evidence of reduced cerebral perfusion pressure during intra- 
cranial pressure monitoring had grade II or III EEGs emphasises that poor cerebral perfusion pressure or raised intracranial pressure are not the only factors producing cerebral damage (though periods of raised pressure with poor cerebral perfusion before monitoring cannot be excluded in all cases). Indeed, when the first EEG shows electrocerebral silence, invasive monitoring of intracranial pressure is contraindicated.

This study shows that serial EEGs are important as a guide to outcome in patients without evidence of poor cerebral perfusion pressure, and are of value in cases with unreliable monitoring. In nine patients with borderline cerebral perfusion pressure measurements the EEG seemed to be of value in assessing whether there had been irreversible impairment of cerebral function. Monitoring with a continuous single channel signal processed EEG (Cerebral Function Analysing Monitor $)^{5}$ may have a place in the identification of compromised cerebral function caused by episodes of raised intracranial pressure. This is currently being studied in this unit, though in the comatose patient single channel monitoring can never replace serial multichannel EEGs.

The EEG reflects the overall picture of multiple factors that may alter cerebral function including damage from failure of other organs, and the cumulative effects of periods of poor cerebral perfusion pressure. ${ }^{6}$ It is not surprising therefore that the EEG has a valuable role in the prognosis of children with the likelihood of raised intracranial pressure complicating non-traumatic coma. Although some recent studies suggested that evoked potentials are more useful and reliable than EEGs as prognostic indicators in comatose patients, ${ }^{78}$ evoked potentials only give information about specific pathways and cortical areas and may therefore be misleading when used in isolation. Studies using evoked potentials may, however, be helpful in supplementing the EEG findings-for example, somatosensory evoked potentials are particularly useful in patients receiving barbiturates, and brain stem auditory evoked potentials give information about brain stem function unobtainable from the EEG. ${ }^{9}$ The value of studies using both EEG and evoked potentials in a larger group of infants and children with non-traumatic coma will be the subject of a further report.

RCT was supported by the Child Health Research Appeal Trust.

\section{References}

1 Tasker RC, Matthew DJ, Helms P, Dinwiddie R, Boyd S. Monitoring in non-traumatic coma. Part I: invasive intracranial measurements. Arch Dis Child 1988;63:888-94.

2 Aoki Y, Lombroso CT. Prognostic value of electroencephalography in Reye's syndrome. Neurology 1973;23:333-43.

3 Janati A, Erba G. Electroencephalographic correlates of neardrowning encephalopathy in children. Electroencephalogr Clin Neurophysiol 1982;53:182-91.

4 Pampiglione G, Harden A. Resuscitation after cardiocirculatory arrest. Lancet 1968;i:1261-5.

${ }^{5}$ Maynard DE, Jenkinson JL. The cerebral function analysing monitor. Anaesthesia 1984;39:678-90.

${ }^{6}$ Markand ON. Electroencephalography in diffuse encephalopathies. J Clin Neurophysiol 1984;1:357-407.

7 Goff WR, Shaywitz BA, Goff GD, et al. Somatic evoked potential evaluation of cerebral status in Reye syndrome. Electroencephalogr Clin Neurophysiol 1983;55:388-98.

${ }^{8}$ De Meirleir LJ, Taylor MJ. Prognostic utility of SEPs in comatose children. Pediatric Neurology 1987; 3: 78-82.

${ }^{9}$ Lutschg Y, Pfenninger J, Ludin DP, Vassella F. Brainstem auditory evoked potentials and early somatosensory evoked potentials in neurointensively treated comatose children. Am J Dis Child 1983;137:421-6.

Correspondence to Dr S Boyd, Department of Neurophysiology, Hospital for Sick Children, Great Ormond Street, London WC1N 3JH.

Accepted 5 February 1988 